\title{
Télescope
}

Revue d'analyse comparée en administration publique

\section{Sûreté, sécurité civile et mesures d'urgence au sein du système canadien de gouvernance multiniveau}

\section{Christian Leuprecht et Todd Hataley}

Volume 19, numéro 1, hiver 2013

La gouvernance multiniveau

URI : https://id.erudit.org/iderudit/1017157ar

DOI : https://doi.org/10.7202/1017157ar

Aller au sommaire du numéro

Éditeur(s)

L'Observatoire de l'administration publique

ISSN

1929-3348 (numérique)

Découvrir la revue

Citer cet article

Leuprecht, C. \& Hataley, T. (2013). Sûreté, sécurité civile et mesures d’urgence au sein du système canadien de gouvernance multiniveau. Télescope, 19(1), 176-193. https://doi.org/10.7202/1017157ar
Résumé de l'article

Dans la panoplie de mécanismes de l'appareil gouvernemental moderne, aucune relation n'est plus semée d'embûches que celle qui existe entre le gouvernement et la société qu'il faut protéger à la fois des menaces extérieures et des troubles internes. La différenciation horizontale et verticale de diverses valeurs collectives, préférences et intérêts a donné lieu à une décentralisation asymétrique qui est le propre de la gouvernance multiniveau des mesures de sécurité, de protection civile et d'urgence au Canada. Cet article trace l'évolution constitutionnelle du système à travers les trois ordres de gouvernement et illustre son fonctionnement à l'aide de deux études de cas. L'effet de décentralisation et d'asymétrie et le désalignement constitutionnel entre les premiers intervenants aux niveaux municipal et provincial par rapport à la capacité d'intervention du fédéral rendent indispensable la collaboration entre les divers paliers. 


\title{
SÛRETÉ, SÉCURITÉ CIVILE ET MESURES D'URGENCE AU SEIN DU SYSTÈME CANADIEN DE GOUVERNANCE MULTINIVEAU
}

\author{
Par Christian Leuprecht, Professeur agrégé, Département de science politique, \\ Collège militaire royal du Canada • christian.leuprecht@rmc.ca \\ Et Todd Hataley, Professeur associé, Département de science politique, Collège \\ militaire royal du Canada • todd.hataley@rmc.ca
}

\begin{abstract}
RÉSUMÉ Dans la panoplie de mécanismes de l'appareil gouvernemental moderne, aucune relation n'est plus semée d'embûches que celle qui existe entre le gouvernement et la société qu'il faut protéger à la fois des menaces extérieures et des troubles internes. La différenciation horizontale et verticale de diverses valeurs collectives, préférences et intérêts a donné lieu à une décentralisation asymétrique qui est le propre de la gouvernance multiniveau des mesures de sécurité, de protection civile et d'urgence au Canada. Cet article trace l'évolution constitutionnelle du système à travers les trois ordres de gouvernement et illustre son fonctionnement à l'aide de deux études de cas. L'effet de décentralisation et d'asymétrie et le désalignement constitutionnel entre les premiers intervenants aux niveaux municipal et provincial par rapport à la capacité d'intervention du fédéral rendent indispensable la collaboration entre les divers paliers.
\end{abstract}

\begin{abstract}
Across the full array of the machinery of modern democratic government, no relationship is more fraught with difficulty than that between government and the society it needs to protect from both external threat and internal disorder. Horizontal and vertical differentiation of diverse territorial and non-territorial community values, preferences, interests and values has given rise to the asymmetric decentralization that is the hallmark of the administration of multi-level security governance in Canada. This article maps the system's historical and constitutional roots across three levels of government. It also uses two case studies to illustrate the workings of this system from the angles of: 1) the compound effect of decentralization and asymmetry; and 2) the constitutional misalignment involving, on the one hand, the first responders working at the municipal and provincial levels and, on the other hand, the surge capacity brought to bear by a federal government largely deprived of a constitutional toolkit that would allow it to constrain rather autonomous provinces. Viewed as a whole, this situation compels adopting an intergovernmental approach whose hallmark is multi-level governance.
\end{abstract}

Pour citer cet article : Leuprecht, C. et T. Hataley (2013). " Sûreté, sécurité civile et mesures d'urgence au sein du système canadien de gouvernance multiniveau», Télescope, vol. 19, n 1, p. 176-193.

U ne large part des études portant sur la gouvernance multiniveau s'intéressent

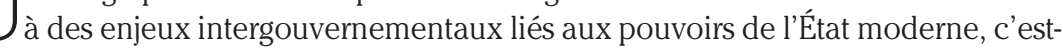
à-dire aux pouvoirs exécutif, législatif, administratif et judiciaire. Toutefois, cette littérature en ignore un cinquième relatif à la paix et à la stabilité du système de gouvernance multiniveau : la protection civile pour assurer la sécurité de la population. Au Canada, le système de sécurité civile est, d'une part, décentralisé et 
suffisamment flexible pour répondre aux diverses exigences et, d'autre part, assez centralisé pour profiter de la coopération intergouvernementale si besoin est. Avec un niveau relativement standardisé de prestation de services, il peut non seulement mener à bien ses tâches quotidiennes, mais également répondre aux besoins lors de périodes de crise et d'urgence. Cet article esquisse d'abord les contours constitutionnels et historiques du système de sécurité publique au Canada. Il examine ensuite la structure institutionnelle en mettant l'accent sur la répartition des pouvoirs et des responsabilités des différents ordres de gouvernement (fédéral, provincial et municipal) et en portant une attention particulière aux changements qui se sont produits à la suite des événements du 11 septembre 2001. Enfin, nous terminons avec les défis de la gouvernance multiniveau liés à la sécurité au Canada, soit l'effet de décentralisation et d'asymétrie ainsi que le désalignement constitutionnel entre les premiers intervenants des paliers municipal et provincial par rapport à la capacité limitée d'intervention du fédéral.

Le gouvernement du Canada a adopté une approche " tous risques " en matière de sécurité civile et de gestion des urgences. Il définit ces termes dans son mandat qui stipule qu'il est responsable de " travailler à la sécurité du Canada sur tous les plans, allant des catastrophes naturelles aux crimes et au terrorisme " (Sécurité publique Canada, 2013). Toutefois, cette définition ne clarifie pas l'étendue des responsabilités du fédéral. Le fait que les deux fonctions relèvent du même ministère suggère que le gouvernement reconnaît un lien entre la sécurité publique et la gestion des urgences et entre les personnes et les programmes visés par son mandat.

En raison du haut degré de décentralisation de la fédération canadienne, la majeure partie des interventions de première ligne, que ce soit en termes de législation ou de gestion des ressources, relèvent des provinces. Constitutionnellement, les municipalités canadiennes ne disposent pas de leur propre champ de compétence. Un exemple du fédéralisme dualiste est illustré par la prestation équitable de la sécurité civile au Canada qui exige manifestement une étroite coopération intergouvernementale. Les ministres se réunissent régulièrement afin de discuter de questions de santé, par exemple, mais de telles consultations dans le secteur de la sécurité publique demeurent inexistantes. Jusqu'à maintenant le manque relatif de coopération n'a pas été mis en évidence, car le système n'a pas réellement été mis à l'épreuve, par exemple les Jeux olympiques de 2010 à Vancouver se sont passés sans problème. En revanche, l'épidémie de SRAS en 2003 a mis en évidence un manque de confiance et de communication entre le fédéral et l'Ontario; à la suite des inondations dans la Vallée-du-Richelieu, de nombreux résidents se sont sentis négligés par le gouvernement fédéral; la tempête de verglas de 1998 a plongé de larges zones urbaines de l'Ontario et du Québec dans le noir; le même scénario s'est produit en 2003 après la panne de courant qui a paralysé le nord-ouest de l'Ontario pendant des jours. Peut-on alors affirmer que le Canada assure la sécurité civile à travers les différents ordres de gouvernement et que le système fonctionne très bien? Ou la fédération canadienne a-t-elle tout simplement été chanceuse et ces récentes expériences ont exposé des défauts qui remettent en question une structure fédérale vieillissante et mal adaptée aux défis de sécurité civile du XXI ${ }^{\mathrm{e}}$ siècle? 


\section{- LES CONTOURS CONSTITUTIONNELS}

L'accord de partage du pouvoir conclu après 1867 a donné au fédéral le contrôle sur les questions de sécurité de l'État et de son intégrité territoriale, tout en cédant les affaires locales aux provinces (et aux municipalités en tant qu'appendices unitaires des provinces). L'après-11 septembre 2001 a entraîné une multiplication de mandats sous-financés ou non financés à l'échelle municipale en raison de nouvelles exigences imposées par les provinces sans allocation de ressources complémentaires et de la non-participation du fédéral dans les affaires municipales (sauf en financement d'infrastructure) afin de respecter l'autonomie provinciale (Henstra, 2003). Cette approche risque d'être inefficace dans un pays dont les six plus grandes métropoles sont plus peuplées et ont un poids économique plus important que quatre des dix provinces du Canada.

Le préambule de l'article 91 de l'Acte de l'Amérique du Nord britannique de 1867 autorise le Parlement fédéral « à faire des lois pour la paix, l'ordre et le bon gouvernement ». Selon cet article, les éléments qui relèvent de la compétence du gouvernement fédéral incluent le droit pénal, les mesures d'urgence, la sécurité nationale, la politique sur les crimes violents et la prérogative de l'exécutif politique à l'égard de la politique étrangère et de la défense. En revanche, selon l'article 92 , tous les enjeux de nature " locale " sont du ressort des gouvernements provinciaux. Ce type de flexibilité garantit des solutions locales aux problèmes locaux de sécurité civile.

En théorie, les deux articles délimitent les frontières du champ de compétence. Dans la pratique, le régime actuel est marqué par une absence d'entente sur les rôles et responsabilités des différents ordres gouvernementaux (Fédération canadienne des municipalités, 2008, p. 5). Devant cette ambiguïté, il faut recourir aux mécanismes intergouvernementaux pour résoudre la problématique suivante : qui paie et qui fournit les services? Le fédéral, par exemple, a la capacité législative de standardiser certaines pratiques de sécurité au niveau local. Selon l'article 91, le Parlement du Canada décide du Code pénal, mais l'administration de la justice relève des pouvoirs de la province. La législation sur les armes à feu, qui fait partie du Code criminel depuis 1892, a longtemps été considérée comme une affaire locale (en particulier dans les régions rurales et nordiques) et ne devait pas être réglementée au niveau fédéral.

Malgré la continuation des principes fondamentaux de la Constitution canadienne depuis 1867, la législation fédérale a dû s'adapter. La législation antiterroriste et la Loi sur la gestion des urgences (2007) sont des exemples contemporains de gestion d'une entente entre le fédéral et le provincial pour partager des responsabilités dans le domaine de la sécurité civile. La structure fédérale des mesures d'urgence reconnaît l'autorité et la capacité des provinces de faire face aux désastres jusqu'à ce que le gouvernement provincial demande de l'aide au fédéral ou jusqu'à que ce dernier estime que le désastre a des conséquences fédérales; il ne peut intervenir que dans ces deux circonstances. 


\section{- L'HISTORIQUE DE LA SÉCURITÉ CIVILE AU CANADA}

Le retrait des troupes britanniques, l'exigence d'assumer la responsabilité de sa propre défense et les menaces qui pesaient sur l'intégrité territoriale incitaient fortement le Canada à s'unifier. L'instabilité liée à la guerre civile aux États-Unis risquait de déborder au Canada et les Canadiens, en raison de l'idéologie américaine du destin manifeste, craignaient une réelle invasion des États-Unis. Des raids de radicaux irlando-américains (appelés les fenians) traversant la frontière vers le Canada ont suscité l'intérêt local, mais concourraient en même temps à élargir les rangs de la milice canadienne, contribuant ainsi à unifier et à définir la coopération de sécurité entre le fédéral et le local (Haglund, 2008). De même, la création de la Police à cheval du Nord-Ouest (maintenant la Gendarmerie royale du Canada - GRC) était une réponse à la violence engendrée par les ravages du whisky vendu par les commerçants américains dans l'Ouest canadien.

Dépourvu d'une tradition militaire, limité par des fonds insuffisants pour payer une armée et aux prises avec la tâche impossible de rendre sécuritaire la frontière entre le Canada et les États-Unis, le gouvernement canadien n'avait d'autre choix que d'invoquer un système de responsabilités partagées. Avec un système local de sûreté déjà en place sous la forme de polices locales et de milices, il était politiquement opportun de déléguer, en grande partie, les coûts principaux en matière de sécurité civile aux gouvernements provinciaux, mais également de maintenir un contrôle législatif sur les pouvoirs exécutés au niveau provincial. Cette solution atténuait aussi les préoccupations des provinces au sujet de l'empiètement du fédéral - soucis qui trouvent leurs origines en Ontario, mais qui seront bientôt partagés par le Québec.

Â l'époque, le développement d'une milice indépendante canadienne était considéré par les hommes d'État canadiens comme un moyen d'unifier les divers intérêts des Canadiens anglais et français (Morton, 1999). Le modèle de la milice canadienne, basé grosso modo sur l'organisation de la milice américaine, visait la création d'unités militaires localisées, sensibles aux enjeux tels que la langue, la religion, la classe et même les styles traditionnels d'uniformes, mais contrôlées de manière centralisée. Après avoir constaté les effets délétères des milices contrôlées localement selon la Constitution américaine, le Canada, contrairement aux États-Unis, n'a pas subordonné le contrôle des milices; par conséquent, la milice était sous le pouvoir du gouvernement fédéral. Afin d'encourager les provinces à ne pas mettre en place leurs propres milices, des entités provinciales, y compris des municipalités, ont eu carte blanche pour demander de l'aide au fédéral en cas d'urgence ${ }^{1}$. Toutefois, les réponses ainsi que les effectifs étaient toujours à la discrétion du pouvoir exécutif fédéral et des Forces canadiennes. Par conséquent, à différentes reprises au fil des ans, les provinces et les municipalités ont eu l'impression d'être abandonnées quant à la promptitude, l'ampleur et/ou la durée des déploiements.

Cette pratique de longue date a été récemment limitée lorsque le gouvernement fédéral a unilatéralement modifié la loi. Les demandes d'aide au pouvoir civil doivent désormais provenir d'un premier ministre d'une province. 
Avant 1867, la sécurité civile, comprenant en grande partie la police et les pompiers, relevait d'une responsabilité collective, à la base des traditions anglaise et française importées au Canada en provenance d'Europe. La première tentative du gouvernement fédéral d'établir une police nationale date de 1868 lorsque le gouvernement du Canada a créé la police du Dominion. Initialement, la police fédérale se composait uniquement d'une douzaine d'hommes, dont la tâche principale était la protection des édifices fédéraux à Ottawa. Le mandat a été élargi pour inclure la protection d'autres structures fédérales comme les chantiers navals. La police fédérale a été absorbée par la GRC en 1920.

L'" autre " service fédéral de police a été créé en 1873 en réaction à l'anarchie et à la violence dans les territoires de l'Ouest canadien. Nommée à l'origine la Police à cheval du Nord-Ouest, cette force avait comme plan initial de patrouiller sur le territoire qui comprend aujourd'hui le Manitoba, la Saskatchewan et l'Alberta. L'entrée du Manitoba dans la Confédération en 1870 signifiait que le gouvernement fédéral n'avait plus le droit de faire intervenir la police dans cette zone. Une force de police provinciale du Manitoba a été créée avec des fonds fédéraux. À partir de l'entrée de la Saskatchewan et de l'Alberta dans la Confédération en 1905, la Police royale à cheval du Nord-Ouest (comme elle se nommait désormais) s'occupait des services de police des nouvelles provinces sur une base contractuelle. Cet arrangement a duré jusqu'en 1916, date à laquelle les deux provinces ont instauré leurs propres forces de police. En 1928, soumise à des contraintes financières, la Saskatchewan a de nouveau eu recours aux services de la GRC et l'Alberta a fait de même en 1932 (Marquis, 1993). De nos jours, les deux provinces continuent de faire appel à la GRC pour veiller au maintien de l'ordre dans leur province (bien que certaines grandes municipalités disposent de leurs propres services de police qui, à toutes fins utiles, ont le même mandat que leurs homologues provinciaux). En revanche, l'Ontario et le Québec maintiennent leurs propres forces de police provinciales depuis longtemps ${ }^{2}$. Les deux provinces ont une longue tradition de services de police municipaux antérieurs à 1867. Le maintien de l'ordre en soi (par opposition à l'application des lois pénales, par exemple) n'a pas été effectivement réglementé par l'Acte de l'Amérique du Nord britannique. Néanmoins, quelques années après 1867, les deux provinces mettaient en place les bases des structures rudimentaires de police qui ont mené à la création de la Police provinciale de l'Ontario (PPO) et de la Sûreté du Québec (SQ).

L'asymétrie marque le fédéralisme canadien, y compris la prestation des services de police : une police fédérale (la GRC) qui offre également des services de police dans huit des dix provinces, ainsi que dans certaines municipalités sur une base contractuelle. Les municipalités de l'Ontario et du Québec peuvent conclure un contrat avec la province pour obtenir des services de police au niveau municipal, au lieu de mettre en place leur propre force de police (ce qui est souvent

\footnotetext{
2 En vertu de son nom, la Royal Newfoundland Constabulary est souvent perçue comme une force provinciale. Dans la pratique cependant, elle est plus proche d'une force supramunicipale qui survole les grandes communautés urbaines de Terre-Neuve : St. John's, Mount Pearl et les communautés voisines de la péninsule nord-est d'Avalon, Corner Brook, Churchill Falls et l'ouest du Labrador, y compris Labrador City. La GRC supervise le reste de la province.
} 
attrayant pour les petites villes en raison des économies d'échelle). La GRC intervient aussi sur certaines réserves autochtones, et 168 accords de service sont actuellement en vigueur avec 408 communautés autochtones. Cette relation avec les réserves autochtones est importante pour notre propos puisque la capacité des communautés autochtones à jouir d'une autonomie sur certains aspects de la prestation des services est une manifestation du fédéralisme non territoriale, pluraliste (Bauböck, 2001; Karmis, 2006). Environ 77 \% de la population canadienne habite dans des communautés ayant leurs propres départements de police, $15 \%$, dans des communautés desservies par la GRC sur une base contractuelle, 6,5\% dans des communautés gérées par des forces de police provinciales et $0,5 \%$ dans des communautés disposant d'un service de police autochtone.

Le Canada souligne qu'il n'existe pas, de prime abord, de raison pour une approche symétrique de la sécurité civile. Au contraire, différentes communautés requièrent des approches différentes. Que l'Ontario et le Québec aient choisi de créer leur propre corps policier provincial n'est pas seulement lié à un héritage historique. Il s'agit des plus grandes provinces de la fédération. Québec, certes, se veut aussi une société distincte et, à ce titre, tente depuis longtemps de maximiser la quantité et l'étendue des services offerts par la province. Pourtant, les structures de service de police provincial du Québec précèdent l'avènement du nationalisme québécois moderne. Cela tend à prouver que la raison initiale d'instaurer un service de police provincial au Québec était plutôt instrumentale : la prestation efficace de services auprès d'une minorité linguistique est difficile si la police n'est pas en mesure de communiquer dans le langage vernaculaire. À l'origine, la GRC était une force anglophone qui a dû s'adapter aux milieux où les francophones étaient les plus nombreux. Environ 4 millions de Québécois ne parlent que le français; les services de police offerts en langue française sont dont indispensables au Québec.

Une logique similaire s'applique en Ontario. L'Ontario est la province qui compte la plus grande communauté francophone à l'extérieur du Québec, et ce, bien qu'au fil des ans l'immigration ait dilué la proportion de la communauté francophone de l'Ontario. Durant la seconde moitié du XIX ${ }^{\mathrm{e}}$ siècle, de grandes parties de l'Ontario, en particulier le long de la frontière avec le Québec ainsi qu'au nord, parlaient presque exclusivement le français. Ainsi, l'Ontario avait besoin d'un service de police avec une capacité linguistique concomitante (à l'époque la GRC n'était qu'une institution anglophone). À ce jour, le français demeure un atout convoité pour les candidats qui postulent à la PPO, l'Ontario étant la seule province du pays à maintenir un ministère des Affaires francophones. En somme, la taille de la population d'un territoire et sa particularité linguistique semblent influencer l'asymétrie au sein de la fédération canadienne.

Les particularités culturelles et linguistiques expliquent aussi pourquoi certaines réserves ont leurs propres services policiers. En revanche, d'autres sont tout simplement trop petites : une communauté de quelques centaines d'habitants qui doit mettre en œuvre sa propre police risque d'affaiblir la règle du droit dans les principes de l'administration impartiale de la justice. D'autres communautés autochtones sont trop divisées pour établir un consensus sur une police locale et 
d'autres encore ont des problèmes de sécurité tellement importants qu'un service de police géré par la communauté serait totalement débordé et incapable de faire face aux défis de sécurité. En d'autres termes, il existe de bonnes raisons pour l'asymétrie.

\section{- LES DISPOSITIFS FÉDÉRAUX DE SÉCURITÉ CIVILE}

Au fédéral, Sécurité publique Canada (SPC) - ministère créé au lendemain des attentats du 11 septembre 2001 afin de centraliser et de coordonner des organisations hétéroclites - a pour mission de « travailler à la sécurité du Canada sur tous les plans, allant des catastrophes naturelles aux crimes et au terrorisme " (Sécurité publique Canada, 2013). Il remplit ce mandat en travaillant en coordination avec d'autres organismes fédéraux, d'autres ordres de gouvernement, les premiers intervenants, les groupes communautaires, le secteur privé et d'autres pays. Exception faite de son personnel ministériel composé de quelque 1000 fonctionnaires, SPC réunit cinq organismes : la GRC, l'Agence des services frontaliers du Canada, le Service canadien du renseignement de sécurité, le Service correctionnel du Canada et la Commission des libérations conditionnelles du Canada. Relèvent également de son portefeuille, des organismes de surveillance opérationnelle ou administrative des comportements individuels et corporatifs, tels que la Commission des plaintes du public contre la GRC, le Bureau de l'enquêteur correctionnel et le Comité externe d'examen de la GRC.

Le tableau suivant illustre la répartition concrète des responsabilités imposées par la séparation constitutionnelle des pouvoirs fédéraux et provinciaux. La section suivante examine ces divisions plus en détail afin d'expliquer les rôles respectifs du fédéral et du provincial dans la protection du Canada. Ces organisations sont conçues pour être compatibles, il sera donc nécessaire de se livrer à des chevauchements afin de mettre en évidence la façon dont elles sont structurées. 


\section{TABLEAU 1 : LA DIVISION DES POUVOIRS DANS L'ADMINISTRATION DE LA} SÉCURITÉ AU CANADA ET AU QUÉBEC

\begin{tabular}{|c|c|c|}
\hline & COMPÉTENCES FÉDÉRALES & COMPÉTENCES PROVINCIALES \\
\hline Ministères & $\begin{array}{l}\text { - Sécurité publique Canada } \\
\text { - Ministère de la Défense nationale }\end{array}$ & $\begin{array}{l}\text { - Ministère de la Sécurité publique } \\
\text { (Québec) }\end{array}$ \\
\hline Agences & $\begin{array}{l}\text { - Gendarmerie royale du Canada } \\
\text { - Service correctionnel du Canada } \\
\text { - Service canadien du renseignement } \\
\text { de sécurité } \\
\text { - Agence des services frontaliers du } \\
\text { Canada } \\
\text { - Commission des libérations } \\
\text { conditionnelles du Canada }\end{array}$ & $\begin{array}{l}\text { - Sécurité civile } \\
\text { - Services correctionnels } \\
\text { - Services policiers } \\
\text { - Sûreté du Québec } \\
\text { - Sécurité incendie } \\
\text { - Commission québécoise des } \\
\text { libérations conditionnelles }\end{array}$ \\
\hline Mandats & $\begin{array}{l}\text { - Crime } \\
\text { - Assistance en cas de catastrophe } \\
\text { sous certaines conditions } \\
\text { - Sécurité frontalière } \\
\text { - Sécurité nationale } \\
\text { - Mesures et atténuation d'urgence } \\
\text { et du crime }\end{array}$ & $\begin{array}{l}\text { - Crime } \\
\text { - Sécurité incendie provinciale } \\
\text { - Secours aux sinistrés } \\
\text { - Élimination des risques } \\
\text { - Sécurité au travail } \\
\text { - Sécurité des transports routiers }\end{array}$ \\
\hline
\end{tabular}

Comme dit précédemment, SPC est le ministère fédéral principal chargé de la coordination des politiques publiques et des programmes de mesures d'urgence. Son mandat est de s'assurer que l'infrastructure essentielle est en place dans le cas d'une catastrophe naturelle ou d'une attaque violente ayant des répercussions nationales. Pour mener à bien ce mandat, SPC remplit deux fonctions principales : la prestation de programmes et l'élaboration de politiques publiques.

SPC propose des programmes dans cinq domaines distincts : les mesures d'urgence, la sécurité nationale, la mise en application des lois, les services correctionnels et la prévention du crime. Dans chacun de ces domaines, SPC a élaboré un certain nombre de programmes qui sont disponibles à l'échelle fédérale et provinciale et, dans certains cas, accessibles au grand public et au secteur privé. Par exemple, dans le cadre de la priorité de la prévention du crime, SPC a mis sur pied le Centre national de prévention du crime qui soutient des initiatives ciblées de prévention de la criminalité et diffuse les meilleures connaissances pratiques. Organisme multiniveau, ce centre travaille en partenariat avec les trois ordres de gouvernement ainsi qu'avec des groupes communautaires et le secteur privé.

Les politiques formulées par SPC reflètent ses cinq priorités, telle la Stratégie nationale de prévention du crime. Définie en 1998, cette stratégie est administrée par le Centre national de prévention du crime en collaboration avec les provinces et, à l'instar du Centre, réunit les mêmes partenaires. SPC n'a cependant pas en soi de capacité à administrer et à exécuter les programmes; à cette fin, il se réfère aux agences qui font partie du portefeuille. 
Le principal organisme prestataire de programmes de sécurité civile à l'échelon fédéral est la GRC, mais il existe néanmoins quatre autres organismes : le Service correctionnel du Canada, la Commission des libérations conditionnelles du Canada, l'Agence des services frontaliers du Canada et le Service canadien du renseignement de sécurité. Le mandat de ce dernier est d'enquêter et de faire rapport sur les activités susceptibles de menacer la sécurité du Canada. En outre, il fournit des évaluations de menaces au gouvernement du Canada et à d'autres organismes fédéraux; il travaille principalement sur le territoire du pays bien qu'il puisse, de manière plus limitée, travailler à l'extérieur. L'Agence des services frontaliers du Canada est responsable de l'ensemble des ports d'entrée au Canada, dont les postes frontaliers terrestres, les points d'entrée dans les terminaux aériens internationaux et les terminaux maritimes. Elle applique les exigences d'entrée aux personnes arrivant au Canada ainsi que les règlements commerciaux. Le Service correctionnel du Canada gère les installations correctionnelles dans lesquelles sont détenus les individus condamnés à deux ans ou plus de prison ferme dans un pénitencier fédéral. Dans le cadre de son mandat, il est chargé des programmes visant à réduire les taux de récidive ainsi que d'une variété de programmes de formation dans les prisons et au sein des communautés. Enfin la Commission des libérations conditionnelles du Canada voit à la prise de décision quant à la libération des prisonniers des prisons fédérales. Dans les provinces qui n'ont pas d'équivalent provincial des prisons provinciales, la Commission agit à titre provincial pour accepter, refuser ou révoquer la libération conditionnelle.

\section{La Gendarmerie royale du Canada}

À l'échelon fédéral, la GRC mène des enquêtes pour faire respecter les lois fédérales telles que la Loi sur les douanes, la Loi sur l'accise, la Loi sur la radiodiffusion, la Loi sur les télécommunications et la Loi sur la corruption d'agents publics étrangers. D'autres lois sont également appliquées par les corps policiers provinciaux et municipaux telles que la Loi réglementant certaines drogues et autres substances et la Loi sur l'immigration et la protection des réfugiés. La GRC est également chargée de la protection des autres ministères fédéraux et des dirigeants nationaux et étrangers. Enfin, les Services nationaux de police de la GRC offrent à d'autres services policiers une formation spécialisée en recherche, en enquêtes médicolégales, en identification.

Huit des dix provinces sont contrôlées à l'échelle provinciale par la GRC bien qu'il s'agisse d'une compétence provinciale. L'arrangement selon lequel une province peut externaliser les obligations policières provinciales au gouvernement fédéral s'appelle le "service de police contractuel ", service qui se distingue des services de police fédéraux bien qu'il s'agisse du même corps de police : le premier est payé par la province, le second par le fédéral. Ces contrats sont négociés et administrés non pas directement par la GRC mais par Travaux publics et Services gouvernementaux Canada qui doit garantir des conditions favorables aux deux parties.

Les provinces ont tout avantage à confier le service de police provincial au fédéral. Premièrement, il y a intérêt à partager les coûts : les provinces, les territoires 
et les villes prennent en charge 70 \% des coûts de la GRC pour gérer les tâches policières provinciales, tandis que le gouvernement fédéral en assume $30 \%$. Les municipalités peuvent aussi faire appel à la GRC, mais cela revient plus cher par habitant pour les municipalités que pour les provinces, car la part fédérale qui était de 50 \% en 1976 a graduellement été supprimée pour tous les contrats municipaux signés après 1992, et ce, bien que le fédéral continue à partager de 10 à $30 \%$ des coûts pour les municipalités pour les contrats antérieurs à 1992 (Fédération canadienne des municipalités, 2008, p. 17). Dans le même temps, la demande pour les services de police a considérablement augmenté (Malm et autres, 2005), ce qui signifie que les réductions de la part du fédéral (ou du provincial) correspondent à une tentative latente de reléguer les coûts à un autre ordre de gouvernement.

Deuxièmement, c'est non seulement un moyen efficace pour la GRC d'établir une présence nationale (tout en allégeant les dépenses fédérales liées à cette entreprise), mais c'est également une méthode pratique pour surmonter des défis entre les provinces. Actuellement, la GRC intervient dans 8 provinces sur 10, dans les 3 territoires, 190 municipalités, 184 communautés autochtones et 3 aéroports internationaux. Les niveaux de satisfaction du service sont honorables : $84 \%$ de répondants à un sondage réalisé en 2011 faisaient confiance à la GRC (Lunney, 2012, p. 441).

Le ministère de la Défense nationale joue également un rôle important en matière de sécurité civile. En principe, les Forces canadiennes ne détiennent pas de mandat de sécurité nationale et n'ont pas d'autorité sur la sécurité civile quotidienne des citoyens canadiens et de leurs biens. Elles sont sollicitées uniquement en cas d'extrême urgence lorsque leur rôle se limite à soutenir la sécurité civile. Les provinces ont alors la possibilité de faire appel aux Forces lorsque les ressources provinciales sont débordées et qu'il y a un risque de répercussions à l'échelle nationale. Même lorsque les Forces canadiennes sont déployées à la demande des autorités provinciales pour faire face à des perturbations civiles, leur présence peut être controversée, comme ce fut le cas au Québec en 1970 lors de la crise d'octobre.

\section{- LES EFFECTIFS PROVINCIAUX DE SÛRETÉ}

Semblable aux dispositifs de sécurité civile du gouvernement fédéral, la sécurité civile provinciale a évolué pour s'adapter aux exigences de chaque province. La portée géographique et démographique des provinces étant beaucoup plus limitée que celle du fédéral, chacune d'elle a élaboré une législation de sécurité civile unique alignée sur ses propres besoins. Les organismes chargés de la sécurité à l'échelle provinciale étant trop variés pour être examinés en détail, quatre cas représentatifs - le Québec, l'Ontario, le Manitoba et la Colombie-Britannique - seront présentés. Chaque exemple décrit le fonctionnement du système de sécurité et montre que la décentralisation de l'administration de la sécurité civile permet aux provinces d'être autonomes pour répondre aux enjeux locaux, tout en ayant accès aux ressources et à l'expertise du fédéral. 


\section{Le Québec}

La principale organisation qui assure la sécurité civile au Québec est le ministère de la Sécurité publique du Québec et son corps de police, la Sûreté du Québec (SQ). Ce ministère se consacre à la prévention du crime, à la sécurité incendie, à la protection civile ainsi qu'aux services correctionnels. De même, il offre des programmes et des orientations à d'autres organismes provinciaux, aux municipalités, au grand public et au secteur privé. Similaire à l'Ontario, le Québec dispose d'arrangements formels et ad hoc entre les services de police opérant dans la province. Le Projet Colisée, une enquête de trois ans qui a fini par conduire à la chute de la famille Rizzuto liée au crime à Montréal, est un excellent exemple de la collaboration officielle possible entre les services de police des trois ordres de gouvernement.

\section{L'Ontario}

La structure de la sécurité civile en Ontario ressemble à celle du gouvernement fédéral en raison du nombre d'agences différenciées chargées de la sûreté. Les responsabilités du principal ministère, le ministère de la Sécurité communautaire et des Services correctionnels, peuvent être classées en trois thèmes principaux : les services correctionnels, les services de police et les services de sécurité civile. Les services correctionnels sont responsables du maintien de probation, des prisons provinciales et de la Commission ontarienne des libérations conditionnelles. Les services de police coordonnent les activités de la Police provinciale de l'Ontario (PPO) et de tous les corps de police municipaux de la province. Enfin, les services de sécurité civile ont pour mandat de coordonner les services entre les pompiers et les services municipaux d'urgence de la province. Ils proposent également des programmes et des services sur les initiatives de sécurité civile aux secteurs public et privé.

La PPO conçoit et propose l'entraînement aux services de police autochtones en Ontario, effectue une campagne de sensibilisation auprès de ses policiers, met sur pied l'équipe provinciale de liaison - organisation à plein temps pour unir les communautés autochtones - et leur donne accès à des services de soutien. La PPO gère également un bureau de police autochtone qui s'occupe du service de police d'une douzaine de communautés autochtones " qui n'ont pas encore exercé leur option de services policiers autogérés, conformément à l'accord relatif aux services policiers des Premières nations " (Police provinciale de l'Ontario, 2009). Bien que le programme des services de police autochtones de l'Ontario soit géré par la PPO (l'article 54 de la Loi sur les services policiers de l'Ontario stipule que le commissaire de la PPO peut nommer des agents de police des Premières nations), la plupart des communautés autochtones n'ont pas d'agents de la PPO étant donné qu'elles bénéficient de leurs propres services de police. Non seulement cette force est-elle plus représentative des communautés qu'elle dessert, mais une formation spécialisée est également nécessaire pour s'assurer que chaque agent est sensibilisé aux enjeux autochtones. 
Bien qu'une répartition claire des responsabilités semble exister entre le fédéral et le provincial dans le domaine de la sécurité civile, la coopération et la coordination quotidiennes des opérations sont la norme. Dans certaines situations, la coordination intergouvernementale est formalisée par un accord. Dans d'autres, la disposition est plus ad hoc. Dans les deux cas, le leadership et le financement sont déterminés d'une situation à l'autre.

\section{Le Manitoba}

Contrairement à l'Ontario et au Québec, le Manitoba n'a pas de ministère centralisé chargé de la sécurité civile et des mesures d'urgence. Étant une province moins peuplée, le Manitoba compte un ministère de la Justice et un ministère de l'Infrastructure, mais ceux-ci ne sont pas centralisés au sein d'une seule organisation. De plus, le Manitoba, n'ayant pas de police provinciale, mandate les services de police de la GRC dans les petites collectivités et les zones rurales. Les grands centres urbains, comme Winnipeg et Brandon, de même que quelques-unes des plus petites communautés rurales ont choisi de créer leurs propres corps de police municipaux, ce qui permet d'assurer un contrôle local sur la sécurité.

Dans le passé, le Manitoba a dû puiser de manière disproportionnée dans les ressources d'urgence du gouvernement fédéral. La répartition démographique de la province complique les évacuations d'urgence. Par exemple, durant l'été 2007, le Manitoba a connu onze tornades, dont une a atteint une force de niveau F5 sur l'échelle de Fujita (Environnement Canada, 2007). Le Manitoba subit aussi des inondations saisonnières. Le débordement de la rivière Rouge en 1997 est considéré comme l'une des plus grandes catastrophes connues au Manitoba : 7000 militaires ont été déployés durant 36 jours pour évacuer plus de 25000 personnes, et plus de 1000 maisons ont été perdues (province du Manitoba, renseignement sur les inondations). À la suite de ces inondations, et avec l'appui du gouvernement fédéral, la province a instauré des contrôles rigoureux pour lutter contre les inondations permettant ainsi d'éviter quelque 10 milliards de dollars de dégâts lors des inondations de 2009 (Henstra et McBean, 2005; province du Manitoba, renseignement sur les inondations). Le cas du Manitoba montre que même les citoyens qui vivent dans des provinces disposant de ressources plus limitées bénéficient d'une gamme complète de protection des gouvernements provincial et fédéral.

\section{La Colombie-Britannique}

En Colombie-Britannique, la sécurité civile fait partie du portefeuille du ministère de la Justice qui gère des domaines traditionnels comme la police, les mesures d'urgence, les prisons ainsi que les services de protection de l'enfance, l'octroi de licences de l'industrie, les services aux tribunaux et automobiles. La plupart des services de police de la province sont pris en charge par la GRC qui s'occupe de l'ensemble des services policiers à l'échelon provincial ainsi que dans 61 zones urbaines (par rapport à 11 services de police municipaux) et gère un corps de police autochtone. Comme dans le reste du Canada, les services d'enquête fédéraux de la GRC interviennent en Colombie-Britannique. 
Afin de réduire les risques de santé associés à la consommation de drogues injectables, la province a établi un programme d'échange de seringues à Vancouver. Le programme Insite fournit des seringues propres aux toxicomanes pour réduire la propagation des maladies liées à l'usage de drogues. Bien que la Cour suprême ait finalement statué en faveur de la province, les cliniques d'Insite restent peu populaires auprès du gouvernement fédéral qui a tenté à plusieurs reprises de fermer les cliniques. Malgré cette opposition, la GRC collabore avec les organisations provinciales pour atteindre les objectifs provinciaux.

Ce survol provincial montre que la gestion de la sécurité civile est profondément asymétrique. Cette asymétrie va au-delà de la disponibilité des ressources pour s'étendre aux différences culturelles, climatiques et linguistiques. Le Canada a beau être un pays pacifique et sécuritaire pour ses citoyens, il fait face aux mêmes problèmes archétypaux que d'autres pays fédérés. Il doit faire face à la consommation de drogues, aux catastrophes naturelles, à la violence familiale et à la menace terroriste. La décentralisation des responsabilités pour assurer une gestion de la criminalité et des mesures d'urgence propre à chaque communauté est une stratégie efficace pour relever ces défis. Surtout, cette décentralisation des responsabilités doit être accompagnée d'une décentralisation des ressources.

\section{- LES DÉFIS D’UNE GOUVERNANCE MULTINIVEAU DE SÛRETÉ AU CANADA}

Dans l'optique intergouvernementale, on constate l'absence d'une approche coordonnée au développement de priorités et d'objectifs entre le fédéral et les provinces (Lunney, 2012, p. 438-439). D'une part, le fédéral insiste pour qu'un seul arrangement soit conclu pour toutes les entités constituantes, et ce, en dépit des différentes exigences des provinces (Lunney, 2012, p. 436). Dans les dix provinces, les ministres respectifs de la sécurité publique (ou leur équivalent) ont la responsabilité de fixer les priorités et les objectifs pour le service de police provincial, tandis que les priorités fédérales sont définies par le ministre fédéral de la Sécurité publique au Parlement dans le cadre des priorités fédérales stratégiques. Cela donne lieu à une déconnexion entre les priorités fédérales et provinciales et amène certaines provinces à réfléchir à leurs propres forces de police provinciales. Tandis que les contrats de police établis entre la GRC et les provinces permettent aux autorités provinciales de demander la révision du programme et d'y participer, les provinces sont peu consultées sur le fonctionnement et la gestion de la GRC (Lunney, 2012, p. 442). Un exemple récent de coordination fédérale-provinciale dans ce domaine est la mise en place par la Colombie-Britannique d'un bureau des enquêtes indépendantes (Independent Investigations Office), dirigé par des civils nommés par la province, qui mène des enquêtes criminelles sur des incidents impliquant des policiers en cas de décès ou de dommage grave ${ }^{3}$. Cet effort aurait été vain s'il n'avait pas intégré la GRC puisqu'aucune autre province ne compte autant de membres de la GRC en termes absolus et relatifs : plus d'un tiers des

3 Des organismes semblables existent en Nouvelle-Écosse, en Ontario, au Manitoba et en Alberta. 
agents de police, c'est-à-dire 6000 sur les 19000 membres opérationnels de la GRC, se trouvent en Colombie-Britannique.

L'asymétrie n'est pas limitée aux relations fédérales-provinciales. Elle est également répandue dans les relations locales-provinciales et locales-fédérales, mettant donc l'accent sur la difficulté d'opérationnaliser le fédéralisme pluraliste, en particulier en ce qui concerne les collectivités autochtones locales. À plusieurs reprises des crises autochtones ont retenu l'attention du grand public : Oka (1993), Lac Gustafsen (1995), Ipperwash (1995), Kashechewan (2005-2006), Calédonie (2006) et Attawapiskat (2011). Elles ont focalisé l'attention sur les questions de contrôle démocratique. Les fonctionnaires risquent d'exacerber l'asymétrie en défendant leurs propres intérêts qui peuvent être en contradiction avec ceux des gouvernements qu'ils sont censés servir (Lindsay, 2009). La relation difficile entre la GRC et l'ancien ministère du Solliciteur général et de son successeur, Sécurité publique Canada, en est un exemple par excellence (d'Ombrain, 1999). Il s'ensuit un système de gouvernement à paliers multiples dont les différents ordres de gouvernement, leurs bureaucraties et les collectivités locales ont des intérêts, des priorités, des préférences et des valeurs qui les poussent à poursuivre des stratégies concurrentes, contradictoires ou inconciliables.

Ces tensions de gouvernance multiniveau sont particulièrement apparentes dans les controverses sur l'allocation des ressources. Les gouvernements provinciaux peuvent être réticents à créer une infrastructure dont la plupart des électeurs ne reconnaissent pas l'importance des mesures préventives. La gouvernance multiniveau peut donc freiner la mise en œuvre rapide d'une législation pour gérer les catastrophes ou la criminalité. Quant au fédéral, le sommet du G20 organisé au Canada en 2010 pour un coût de 1,2 milliard de dollars est un exemple de la méfiance des citoyens à l'égard de la légitimité du gouvernement fédéral en raison des dépenses de sécurité perçues comme un gaspillage. Le rapport de la vérificatrice générale du Canada sur le sommet a confirmé la préoccupation exprimée par les citoyens canadiens et les partis d'opposition sur ce genre de gaspillage (Vérificatrice générale, 2011, chap. 1-2).

Le cas du G20 n'est qu'une illustration des problèmes créés par un système fédéral dont la constitution exclut les municipalités de la structure fédérale-provinciale en les traitant comme des appendices des provinces. Au Canada, en raison d'une construction qui privilégie les relations fédérales-provinciales, les premiers intervenants finissent par être privés de voix et de ressources (Juillet et Koji, 2013). Alors que la capacité de première intervention se situe largement au niveau des collectivités locales, le flux descendant de fonds ne correspond pas aux besoins et aux réalités : en 2006, les municipalités assumaient 56,6 \% du total canadien des dépenses policières et embauchaient 65,8 \% des policiers au Canada (y compris les sous-traitances pour agents de police provinciaux ou de la GRC), les provinces payaient $24,5 \%$ des effectifs (y compris les policiers provinciaux sous contrat) et le gouvernement fédéral, avec la GRC, ne payait que 9,7 \% (Fédération canadienne des municipalités, 2008, p. 4 et 13).

La marginalisation des administrations municipales du lien fédéral-provincial a également un effet sur la standardisation des mesures d'urgence au niveau local 
et/ou provincial. Les gouvernements locaux, en particulier les plus petits, doivent souvent recourir de manière disproportionnée aux intervenants bénévoles comme première ligne de défense (Ferrier, 2008). En outre, les mandats liés à la gestion de situations d'urgence, tels le transport, la faune et les services publics, sont souvent fragmentés dans plusieurs ministères ou organismes. Les défis de coordination horizontale et verticale qui en découlent peuvent poser des problèmes, en particulier avec les premiers intervenants bénévoles et les partenaires du secteur privé comme les sociétés de services publics dont la formation et la conformité aux normes dépendent du temps, de l'argent, de la coopération et du bénévolat.

Des nouveaux défis inhérents à la sûreté au sein de la fédération canadienne se dessinent à l'horizon et bon nombre d'entre eux seront asymétriques (Leuprecht, Hataley et Nossal, 2012). Les changements climatiques mondiaux amplifient les menaces les plus immédiates pour le Canada. Le réchauffement dans le Nord canadien ouvre des passages dans la glace arctique et relance la question de la souveraineté canadienne dans l'Arctique et des mesures de sécurité, de défense et d'urgence sur une large bande de terre peu peuplée, inhospitalière et éloignée des effectifs de sécurité, telles les bases militaires. De même, les conditions météorologiques risquent de devenir plus graves et imprévisibles : les tempêtes autrefois réservées aux climats tropicaux frappent désormais les villes côtières canadiennes. Les prévisions financières suggèrent que les écarts dans les revenus continueront à croître. De telles disparités sont souvent accompagnées d'agitation de la part des citoyens lésés, ce qui risque d'engendrer de l'instabilité régionale. Enfin, le Canada fait face à une menace nouvelle et totalement imprévisible. Dans un climat de sécurité internationale, les organisations terroristes comptent sur des tactiques de surprise pour ravager des cibles civiles. Afin de faire face à ce nouveau type de menace, il faut mettre en place un système intergouvernemental de mesures de sécurité civile et d'urgence qui soit capable d'agir rapidement et efficacement. Cela nécessitera non seulement une coopération accrue, mais aussi l'application judicieuse des leçons apprises ailleurs au sein de systèmes à paliers multiples. Or ni les provinces ni les municipalités n'ont les ressources financières, organisationnelles et de leadership nécessaires; celles-ci sont acquises par le gouvernement fédéral qui craint de s'impliquer et d'empiéter sur les compétences provinciales. Cela donne lieu à un problème d'action collective que les municipalités et les provinces ne peuvent surmonter seules.

\section{- CONCLUSION}

Le système public canadien de mesures de sûreté, de sécurité civile et d'urgence est caractérisé par la décentralisation et l'asymétrie. Cette flexibilité peut être à la fois le plus grand atout et la plus grande faiblesse du système multiniveau canadien : elle permet d'adapter les mesures de sécurité civile et d'urgence aux risques et aux besoins, tout en laissant une latitude considérable aux gouvernements fédéral et provinciaux. Bref, le gouvernement fédéral est le premier à intervenir sur les questions qu'il traite, même si les municipalités sont les premiers intervenants dans la plupart des questions liées aux mesures de sécurité civile et d'urgence (Fédération canadienne des municipalités, 2008; Mukherjee, 2011). 
Parce qu'elles détiennent un grand pouvoir sur leurs revenus fiscaux, qu'elles collectent sensiblement les mêmes impôts que le gouvernement fédéral et qu'elles bénéficient de la péréquation et d'autres transferts importants largement inconditionnels, les provinces exercent une autonomie décisionnelle considérable. Ceci n'est pas le cas pour les relations entre une province et ses municipalités. Les provinces tentent de déléguer les responsabilités liées aux mesures de sécurité publique et d'urgence aux municipalités sans forcément récompenser ces dernières pour leurs nouvelles tâches. Pire encore, les exigences spécifiques associées à des mandats non financés forcent les communautés à concentrer leurs efforts sur les risques spécifiques au lieu d'adopter une approche tous risques susceptible de donner de meilleurs gains en mesure de sécurité civile et d'urgence (Henstra, 2010).

Les lacunes constatées au Canada ne sont pas uniques, elles se trouvent aussi dans d'autres systèmes multiniveaux. Pourquoi donc le système canadien fonctionne-t-il relativement bien? Est-ce parce qu'il est décentralisé et asymétrique? Ou reflète-t-il plutôt un pays dont les échelons infranationaux de gouvernement ont l'autonomie constitutionnelle, les moyens financiers et la capacité conséquente pour appliquer les mesures de sécurité civile et d'urgence au sein d'une culture politique libérale démocratique qui respecte la primauté du droit et encourage la conformité et le bénévolat? Les défis liés à la sécurité et aux mesures d'urgence au Canada sont aussi diversifiés que le pays est grand. Le système canadien habilite les provinces et les localités pour qu'elles puissent répondre aux besoins locaux à l'aide d'un arrangement ad hoc d'employés rémunérés et de bénévoles et d'un afflux potentiel du fédéral. Il fonctionne grâce à un agencement de confiance, de capacité et d'autonomie à l'échelle locale qui permet de refléter les valeurs, les préférences, les priorités et les intérêts locaux, et grâce à une forte culture politique qui concilie le partage du pouvoir en fonction d'un engagement fort à la primauté du droit et l'autonomie sous la forme d'un engagement concomitant par le public canadien de prendre en main leur sécurité.

\section{BIBLIOGRAPHIE}

Bauböck, R. (2001). "Cultural Citizenship, Minority Rights and Self-Government ", dans A. Aleinikoff et D. Klusmeyer (dir.), Citizenship Today: Global Perspectives and Practices, Washington, Carnegie Endowment for International Peace, p. 319-348.

D'Ombrain, N. (1999). "The Federal Government and the RCMP ", Canadian Public Administration, vol. 42, $\mathrm{n}^{\circ}$ 4, p. 452-475.

Environnement Canada (2007). La Tornade à Elie est reclassée à la plus haute cote sur l'échelle des dommages - La première tornade F5 officielle du Canada, www.ec.gc.ca/default. asp ?lang=Fr\&n=714D9AAE-1\&news=4B3DE57E-4967-4B09-98D6-EF974B32D6B5 (page consultée en décembre 2012).

Fédération canadienne des municipalités (2008). Towards Equity and Efficiency in Policing: A Report on Policing, Roles, Responsibilities and Resources in Canada, Ottawa, Fédération canadienne des municipalités. 
Ferrier, N. (2008). Fundamentals of Emergency Management: Preparedness, Toronto, Emond Montgomery Publishers.

Gouvernement du Manitoba (2012). Flood Information, www.gov.mb.ca/flooding/ historical_facts.html (page consultée en décembre 2012).

Haglund, D. (2008). " The Parizeau-Chrétien Version: Ethnicity and Canadian Grand Strategy ", dans D. Carment et D. Bercuson (dir.), The World in Canada: Diaspora, Demography, and Domestic Politics, Montréal et Kingston, McGill-Queen's University Press, p. 92-108.

Henstra, D. (2010). " Explaining Local Policy Choices: A Multiple Stream analysis of Municipal Emergency Management ", Canadian Public Administration, vol. 53, n 2, p. 241-258.

Henstra, D. (2003). "Federal Emergency Management in the US and Canada after 11 September 2001 », Canadian Public Administration, vol. 46, n 1, p. 103-116.

Henstra, D. et G. McBean (2005). " Canadian Disaster Management: Moving Towards a Paradigm Shift? ", Canadian Public Policy, vol. 31, n 3, p. 308-318.

Juillet, L. et J. Koji. (2013). " Reforming the Multilevel Governance of Emergencies: Municipalities and Discursive Politics of Canada's Emergency Management Policy ", dans K. A. H. Graham et C. Andrew (dir.), Canada in Cities: The Politics and Policy of Federal-Local Governance, Montréal et Kingston, McGill-Queen's University Press.

Karmis, D. (2006). « Les multiples voix de tradition fédérale et la tourmente du fédéralisme canadien ", dans A.-G. Gagnon (dir.), Le fédéralisme canadien contemporain : fondements, traditions, institutions, Montréal, Presses de l'Université de Montréal.

Leuprecht, C, T. Hataley et K. R. Nossal (2011). Évolution des menaces transnationales et sécurité frontalière : un nouveau programme de recherche, https://qshare.queensu.ca/ Groups/QCIR/www/Publications/Martellos/Martello37F.pdf?uniq=-gerkw2 (page consultée en décembre 2012).

Lindsay, J. (2009). " Emergency Management in Canada: Near Misses and Moving Targets ", Comparative Emergency Management Book Project, Emmitsburg, MD, United States Federal Emergency Management Institute, http://training.fema.gov/EMIWeb/ edu/Comparative\%20EM\%20Book\%20-\%20Chapter\%20-\%20Emergency\%20 Management\%20in\%20Canada\%20-\%20Near\%20Misses\%20and\%20Moving\%20 Targets.doc (page consultée en décembre 2012).

Lunney, R. F. (2012). " Galloping off in All Directions: An Analysis of the New Federalprovincial Agreement for RCMP Contract Police Services and Some Implications for the Future of Canadian Policing ", Canadian Public Administration, vol. 55, n 3 , p. $433-450$.

Malm, A. et autres (2005). A 30 Year Analysis of Police Service Delivery and Costing: "E" Division, Abbotsford, Centre for Criminal Justice Research, University College of the Fraser Valley, and International Centre for Urban Research Studies (ICURS), Simon Fraser University.

Marquis, G. (1993). Policing Canada's Century: A History of the Canadian Association of Chiefs of Police, Toronto, University of Toronto Press.

Morton, D. (1999). Canada: A Millennium Portrait, Toronto, Dundurn. 
Mukherjee, A. (2011). Avoid Crisis, an Opportunity: Transforming the Toronto Police Service. A Discussion Paper, Toronto, Toronto Police Services Board.

Police provinciale de l'Ontario (2009). Ce qu'on fait, www.opp.ca/ecms/index. php?id=220 (page consultée en avril 2013).

Quigley, K. (2013). «'Man Plans, God Laughs': Canada's National Strategy for Protecting Critical Infrastructure ", Canadian Public Administration, vol. 56, n 1, p. 142-164.

Sécurité publique Canada (2013). Ce que nous faisons, www.securitepublique.gc.ca/abt/ wwd/index-fra.aspx (page consultée en avril 2013).

Sécurité publique Canada (2009). Politique fédérale en matière de gestion des urgences, www.securitepublique.gc.ca/prg/em/fpem-fra.aspx (page consultée en décembre 2012).

Statistique Canada (2011). Les ressources policières au Canada, www.statcan.gc.ca/pub/85225-x/85-225-x2011000-fra.pdf (page consultée en décembre 2012).

Vérificatrice générale du Canada (2011). Rapport sur les dépenses pour les sommets du G8 et G20 de 2010 et Rapport sur le fond d'infrastructure du G8, www.oag-bvg.gc.ca/internet/ Francais/parl_oag_201104_f_35230.html (page consultée en décembre 2012). 